\title{
Research and Development of Two Pendulums Vibration Exciter in Vibration Mill
}

\author{
Yang Xiaolan ${ }^{*}, 1,2$, Liu Jifeng ${ }^{1,2}$, Zhang Luoming $^{2}$ and Wang Zhijin ${ }^{1}$ \\ ${ }^{I}$ College of Mechanical Engineering, Nanjing Institute of Technology, Nanjing, 211112, P.R. China \\ ${ }^{2}$ College of Mechanical Engineering, Huanghe Science and Technology College, Huanghe, 450063, P.R. China
}

\begin{abstract}
To overcome the bottlenecks of powder agglomeration and un-refinement in the ultrafine grinding technology, a two pendulums vibration exciter is designed. Based on the double masses vibration theory, the angel between the two pendulums is adjusted to produce a continuously changing exciting force, which can form high transient vibration intensity and large amplitude. Their transient maximums are 18 and $16 \mathrm{~mm}$ respectively. It can effectively deal with powder agglomeration and anti-powder. Therefore, the grinding efficiency is improved. Achievements have been obtained that the output size of diamond particles has reached $\mathrm{d}(50) \leq 0.27 \mu \mathrm{m}$, but the input size is $\mathrm{d}(50) \leq 10 \mu \mathrm{m}$. In addition, the two pendulums spindle strength is checked. Its strength is completely satisfied in the applications.
\end{abstract}

Keywords: Frequency control, two pendulums, vibration exciter, vibration mill.

\section{INTRODUCTION}

Ultrafine super-hard powder has broad application in areas such as aerospace, precision machinery, optical instrument, automotive engineering and information technology. It is unable for a conventional vibration to deal with the powder agglomeration and anti-powder of super hard material. This paper describes the development of a two pendulums vibration exciter which will constantly produce high transient vibration intensity to solve the problem of powder agglomeration [1].

\section{WORKING PRINCIPLE OF VIBRATION MILL}

Superfine powder preparation device is a type of highly efficient pulverizing equipment. Its working theory is utilizing collision and smashing between the medium in the cylinder and diamond powder to refines the diamond powder into smaller particles [2].

Vibration mill is a device using the principle of vibration. It can effectively conduct fine grinding and super fine grinding. It is consisted of upper mass, lower mass, vibration motor, cylinder, pendulum, main spring, isolator etc. As shown in Fig. (1), the working principle of vibration mill is that the vibration motor drives upper mass and transfers the force to grinding cylinder. There are stainless-steel balls whose diameter are $4 \mathrm{~mm}, 6 \mathrm{~mm}, 8 \mathrm{~mm}, 10 \mathrm{~mm}$ in grinding cylinder so as to produce strong longitudinal vibration. This strong longitudinal vibration contributes on the impact and grinding of material in grinding cylinder, and thereby the purpose is reached of fine grinding and crushing [3,4]. By adjusting magnitude of the eccentric block and changing

*Address correspondence to this author at the College of Mechanical Engineering, Nanjing Institute of Technology, Nanjing, 211112, P.R. China; Tel: +86 02586118256; Fax: +8686118255; E-mail: yxhh001@163.com gradation selection of grinding medium, we can control the particle size of the product and improve productivity.

In many cases, the vibrating system with wide power spectrum and high vibration intensity is demanded to finish some special operations, for instance, the inadequate refinement of particles, agglomeration and anti-crush. The conventional vibrating motor is almost helpless to solve these problems.

When the mill operation, due to grinding medium's the repeated impacting with each other, material particles are crushed by fatigue damage. With the deformation of these particles, new surface develops based on the cracks. After unloading, with the function of molecular force, they 'are healing' again, which forms agglomerates. But when the mutation impact of the weakest spots happens, the highfrequency impacting effect of grinding medium on particles results in the micro crack rapid expanding before 'healing', and these particles will produce macroscopic break [5]. Thus it is the most effective way to conduct fine grinding of solid material with the shock of vibration [6,7].

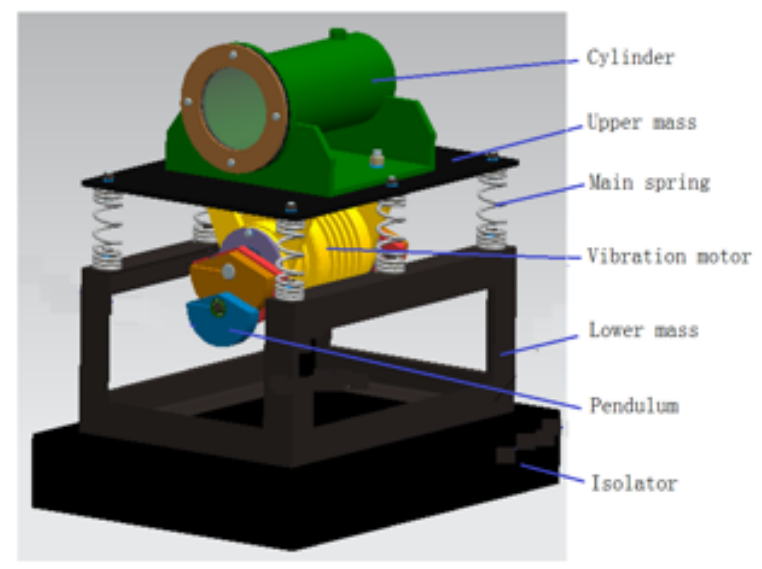

Fig. (1). Vibration mill structure diagram. 


\section{DESIGN OF THE TWO PENDULUMS VIBRATION EXCITER IN NEW VIBRATION MILL.}

To make the grinding cylinder could produce transient high vibration intensity continuously; the pendulum structure can be redesigned. The first level partial block of general vibration motor has been refitted into two partial blocks is called the two pendulums vibration motor. In another word, several partial blocks are series connection. $\mathrm{K}$ is coefficient of series connection, which is decided by the structure. Generally $\mathrm{k}=2$, they are called the two pendulums. Its real image and simulation diagram is shown in Fig. $(\mathbf{2 a}, \mathbf{b})$. The degree of freedom of the mechanism is the partial block's series.

The two pendulums are the main part of the vibration motor which is used to produce exciting force to drive the movement of the vibration mill. According to existing technical experience, the mass of the first level pendulum is $4 \sim 6$ times of the second pendulum's mass. So it could produce exciting vibration force with higher amplitude, more irregular amplitude changing and some mutation amplitude when the pendulums are spinning. These could meet the design requirement of exciting vibration force and transient high vibration intensity $[8,9]$.

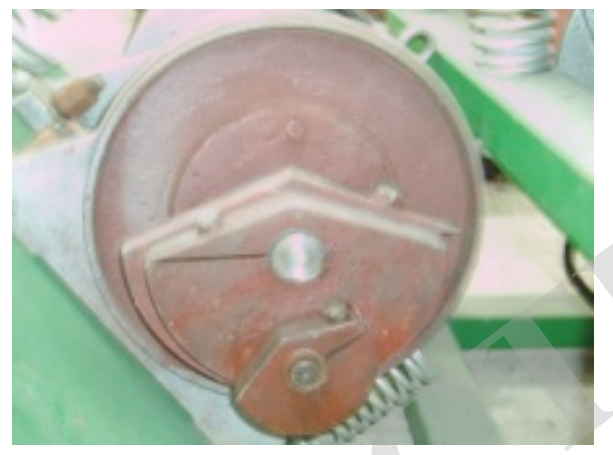

a) Real image

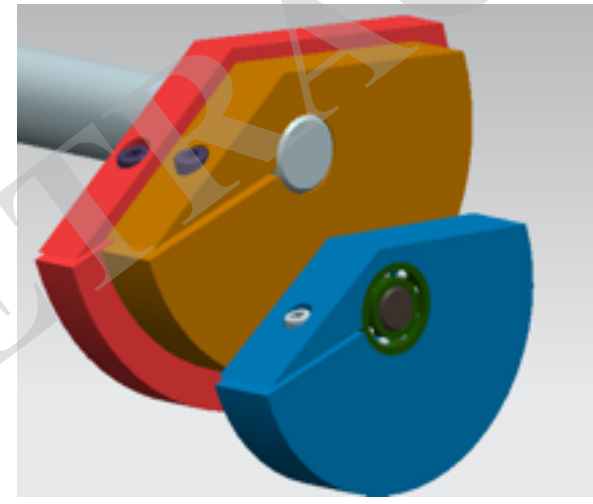

b) Simulation diagram

Fig. (2). Two pendulums.

Adjusting the relative angle between the eccentric block and the fixed block inside, the inertia force and the centrifugal force could be changed. Because of the two pendulums structure, the second pendulum is rolling with insufficient DOF when the motor is spinning. Therefore, it can be seen that the vibration motor output curve is a large amplitude curve without cycle and rule. The vibration mill's amplitude would be larger and the materials in the grinding cylinder would suffer larger impact without cycle and rule, which could avoid the phenomenon that the materials gather together. The materials would be finer grinding. So, it is significant that the eccentric block of the motor and the whole assembly structure of vibration mill should be improved to increase the exciting force and the vibration amplitude [10].

\section{STRENGTH CHECK OF VIBRATION MOTOR SPINDLE AND LIFE-TIME CALULATION OF ROLLING BEARING}

\subsection{Strength Check of Vibration Motor Spindle}

Because of the input torque of the motor, the torsional deformation is occurred on the spindle. The torque is only used to overcome the damping force and friction; it does not to drive the external load. In experience, because of small the power of motor, the torque is very small. To simplify calculation, the torque and the torsional deformation on the spindle could be ignored. Thus, the spindle would suffer from two eccentric forces and two constraining forces from bearing. The force diagrams are shown in Fig. (3).
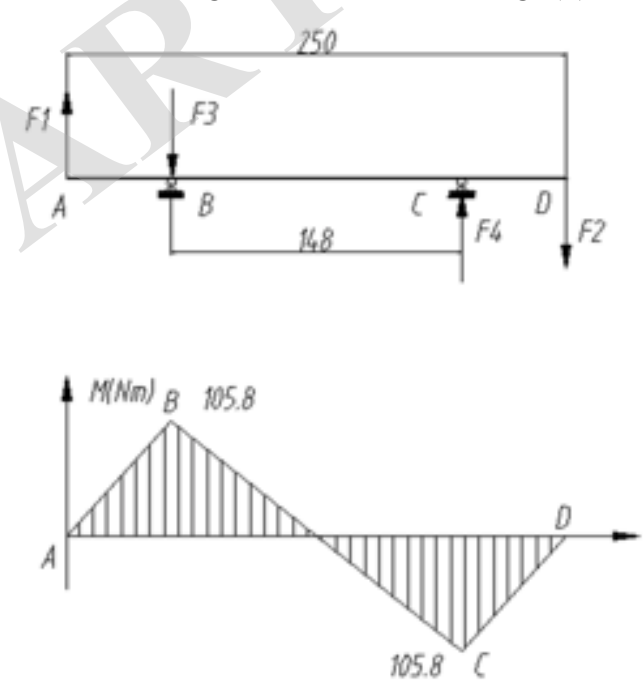

Fig. (3). Force and bending moment diagram of motor spindle.

According to engineering practice, we could get that:

$F_{1}=F_{2}=m r \omega^{2}=1.5 \times 0.035 \times 152.9^{2}=1228 \mathrm{~N}$

where,

$m=1.5 \mathrm{~kg}, r=0.035 \mathrm{~m}, \omega=152.9 \mathrm{rad} / \mathrm{s}$

After the spindle's spinning, $F_{3}$ and $F_{4}$ could be solved by principle of statics in theoretical mechanics and the expression is that:

$\sum F_{y}=0, F_{1}+F_{4}-F_{2}-F_{3}=0$

$\sum M_{B}=0,-F_{1} \overline{A B}+F_{4} \overline{B C}-F_{2} \overline{B D}=0$

Taking $F_{1}$ and $F_{2}$ into the Eqs. 1 and 2, we can get $F_{3}=F_{4}=2074.3 \mathrm{~N} \mathrm{~F}_{3}=\mathrm{F}_{4}=2074.3 \mathrm{~N}$, and the direction is shown in Fig. (3). When the bending moment diagram is drawn, it could be seen that: 
$M_{\max }=105.8 \mathrm{Nm}$

Dangerous section should be at a minimum diameter. According to design parameter of spindle, the minimum diameter of spindle is $20 \mathrm{~mm}$. So, the minimum section modulus in bending is that:

$W_{t \min }=\frac{\pi d_{\min }^{3}}{32}=0.000000785 \mathrm{~m}^{3}$

And the maximum stress which the spindle suffered is that:

$\sigma_{\max }=\frac{M_{\max }}{W_{t_{\text {min }}}}=\frac{105.8}{0.000000785}=134.7 \mathrm{MPa}$

The material of spindle is 45 steel in this design and its yield stress is $\sigma_{s}=350 \mathrm{MPa} \sigma_{\mathrm{s}}=350 \mathrm{MPa}$. If the safety factor is $n=2.0 \mathrm{n}=2.0$, the allowable stress is that:

$[\sigma]=\frac{\sigma_{s}}{n}=175 \mathrm{MPa}$

So, $\sigma_{\max }<[\sigma]$, and the spindle could meet the requirement.

\subsection{Life-time Calculation of Rolling Bearing}

According to the design of spindle, the bearing code (6405 deep groove ball bearing) is taken into the design. Life-time calculation formula could be gotten:

$L_{h}=\frac{16667}{n}\left(\frac{f_{r} C_{r}}{p}\right)^{\varepsilon}$

In the formula, $\mathrm{n}$ is the speed of bearing $\left(\mathrm{n}=\mathrm{n}_{0}=\right.$ 1460r/min);

$\mathrm{f}_{\mathrm{r}}$ is the temperature coefficient $\left(f_{r}=0.95\right)$;

عis the life index ( $\varepsilon=3$ for ball bearing)

$C_{r}$ is the basic rating dynamic $\operatorname{load}\left(C_{r}=14.0 \mathrm{KN}\right)$

$P$ is the equivalent dynamic load and its expression is

$p=f_{p}\left(X F_{\gamma}+Y F_{\alpha}\right)$

In this formula, $f_{p}$ is the impact load coefficient, $\mathrm{f}_{\mathrm{p}}=$ 1.1.

$F_{r}$ and $F_{\alpha} \quad \mathrm{F}_{\gamma}$ andF $\mathrm{F}_{\alpha}$ are radial load and axial load respectively, and the deep groove ball bearing could only suffer the radial load, so $F_{r}=2074.3 \mathrm{~N}, F_{\alpha}=0$.

The $X$ and $Y$ are radial dynamic load coefficient and axial dynamic load coefficient respectively. The deep groove ball bearing could only suffer the radial force, so $x=1, y=0$.

Taking all the data into Eq.6, it could be that:

$L_{h}=\frac{16667}{n}\left(\frac{f_{r} C_{r}}{P}\right)^{\varepsilon}=6565 h$
If the vibration mill works in two-platoon plan daily and it works 340 days a year, the life of the bearing would be 1.2 year. It meets the requirement of production.

\section{GRINDING TEST BASED ON FREQUENCY CONTROL}

Frequency variable sine curve test was designed to find out which speed changes can produce a certain frequency of vibrational state including high vibration intensity and high amplitude. Because vibration intensity and amplitude can be calculated by displacement and acceleration, we can get change of vibration intensity and amplitude by builtin program testing acceleration image of point on vibrating body.

Frequency control for two pendulums vibration mill is implemented according to variable sine curve. The period of sine curve is set to $30 \mathrm{~s}$, step number 300 . Variable speed piecewise function is $600+180 \sin (2 \pi \mathrm{t}), 600+270 \sin (2.5 \pi \mathrm{t})$, $600+360 \sin (3.33 \pi \mathrm{t}), \quad 600+450 \sin (5 \pi \mathrm{t}), \quad 600+540 \sin (10 \pi \mathrm{t})$, $600+450 \sin (5 \pi \mathrm{t}), \quad 600+360 \sin (3.33 \pi \mathrm{t}), \quad 600+270 \sin (2.5 \pi \mathrm{t})$ and $600+180 \sin (2 \pi \mathrm{t})$. Each function is run $2 \mathrm{~s}$. In laboratory, it uses the vibration mill which cylinder type is $\phi 200 \times 400 \mathrm{~mm}$ as a prototype, puts the diamond powder particles which average particle size are $10 \mu \mathrm{m}$, and operates total 100 hours. After the test, amplitude and vibration intensity change curves are shown as Figs. $(4,5)$, and their transient maximum are $16 \mathrm{~mm}$ and 18 respectively, and large amplitude and high vibration intensity have been obtained.

\section{THE TEST RESULTS TO DETECT}

Using BT-9300H laser particle size analyzer detects the particle size distribution of the grinded material. Electron microscope scanning images is shown in Fig. (6), and The percentage of powder particles the cumulative size distribution is as shown in Fig. (7). The particle size distribution is defined as a percentage of the volume of a certain level of powder occupied the total sample volume. Particle size distribution is shown as Table 1. We can see the average particle size $\mathrm{d}(50) \leq 0.27 \mu \mathrm{m}$. It achieved significant refinement particle effects.

The existing technology of first pendulum excitation motor just can change vibration intensity and amplitude slightly. Though this vibration mill can always get a certain vibration intensity and amplitude, because of its small amplitude, it's easy to make ultrafine powder agglomeration, and not conducive to grinding powder. When two pendulums vibration mill variable sine curve frequency control is adopted, vibration intensity and amplitude fluctuation was bigger than that with evenly speed and its amplitude can be increased nearly two times. Amplitude and vibration intensity change alternately no rule, which can easily break the agglomeration of powder, and make low-energy area reduced and useful work increase, so as to achieve refinement particles. 


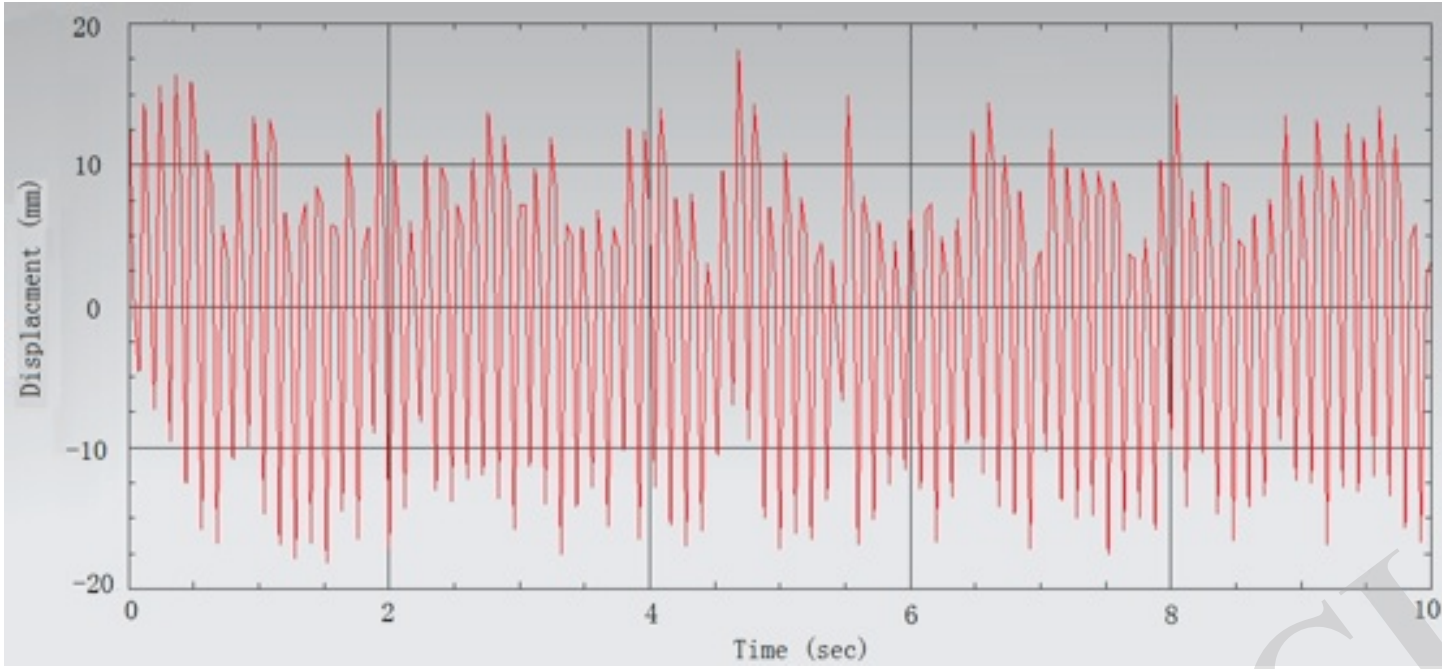

Fig. (4). Amplitude hange curve.

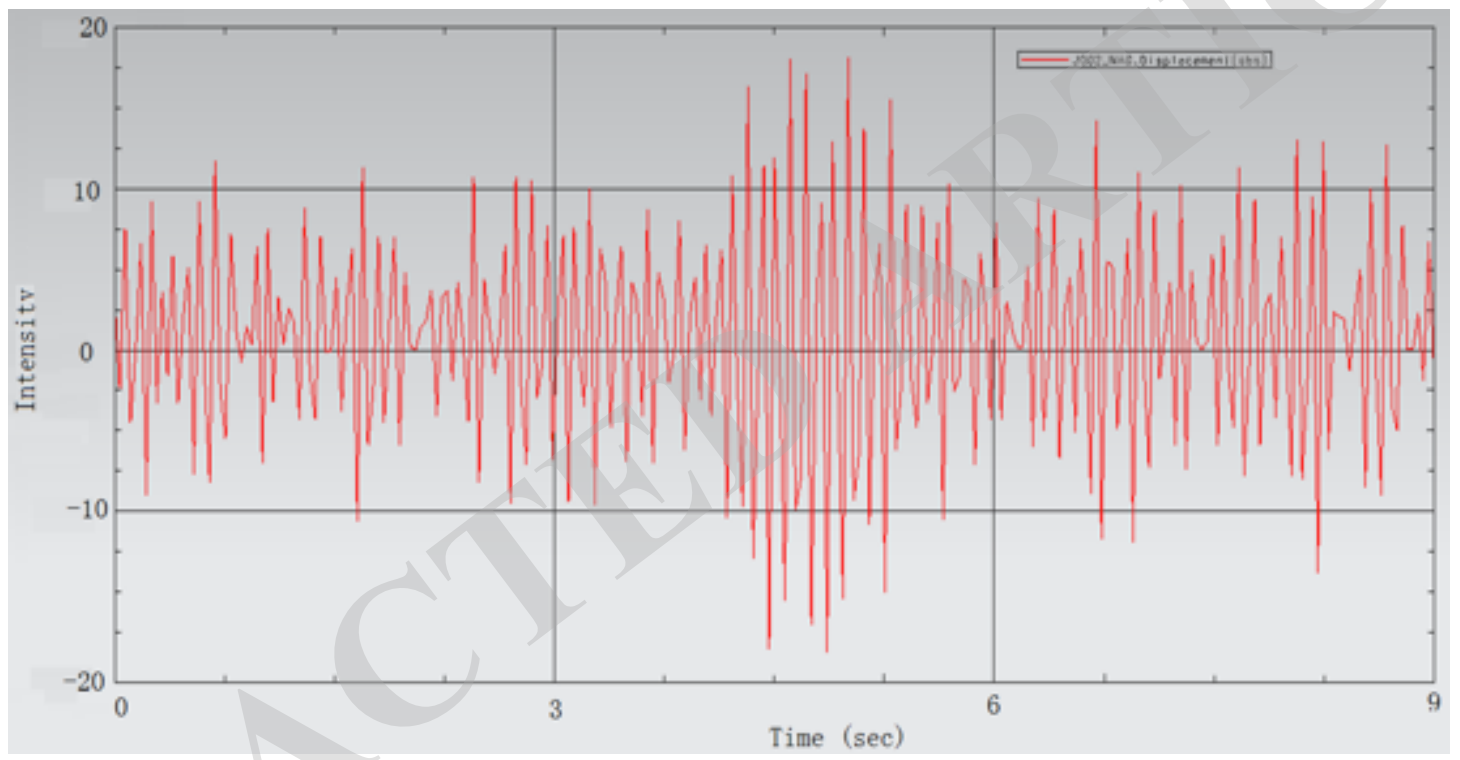

Fig. (5). Vibration intensity change curve.

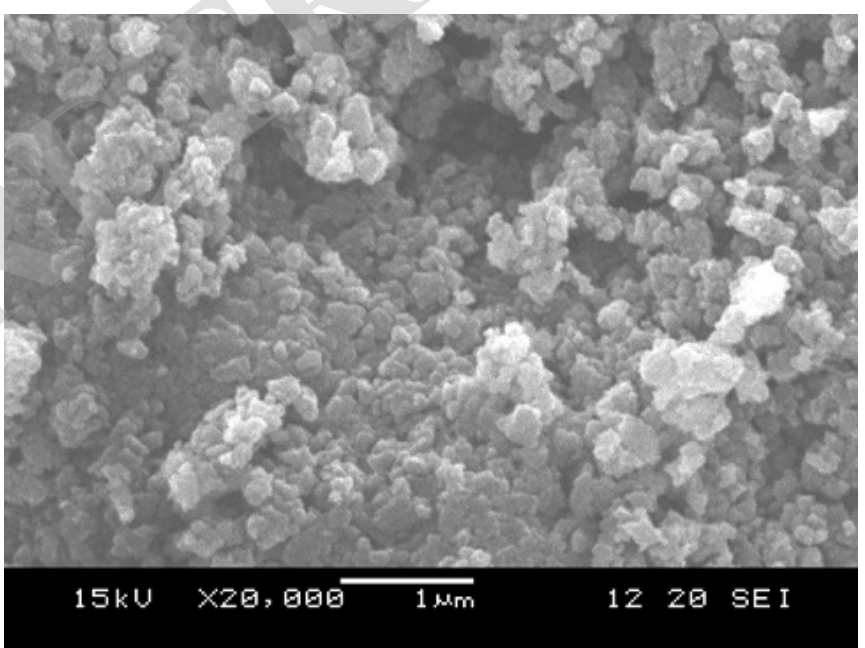

Fig. (6). Electron microscope scanning images.

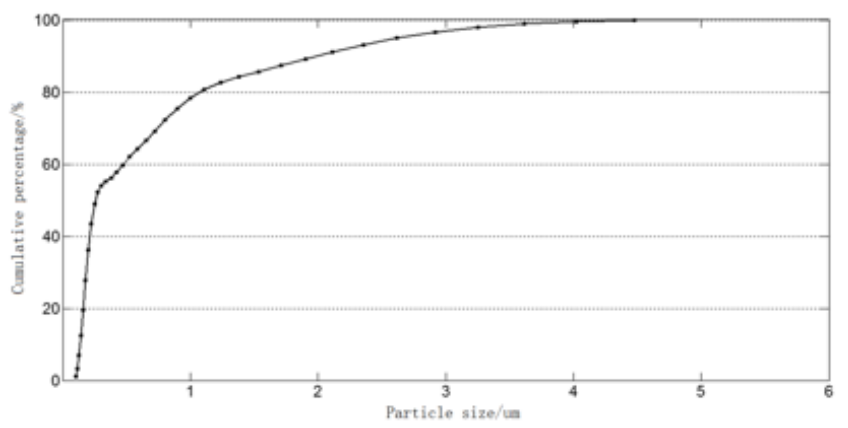

Fig. (7). The percentage of particles the cumulative size distribution.

\section{CONCLUSION}

1) The newly developed two pendulums vibration exciter can constantly change the exciting force by adjusting the angle between two pendulums, so that transient high vibration intensity is produced. The 
Table 1. Particle size distribution.

\begin{tabular}{c|c|c} 
Particle size $(\mu \mathrm{m})$ & Interval $(\%)$ & Accumulation $(\%)$ \\
\hline $0.10-0.11$ & 1.01 & 1.01 \\
\hline $0.11-0.12$ & 2.17 & 3.18 \\
\hline $0.12-0.14$ & 3.78 & 6.96 \\
\hline $0.14-0.15$ & 5.48 & 12.44 \\
\hline $0.15-0.17$ & 7.12 & 19.56 \\
\hline $0.17-0.19$ & 8.26 & 27.82 \\
\hline $0.19-0.21$ & 8.36 & 36.18 \\
\hline $0.21-0.24$ & 7.3 & 43.48 \\
\hline $0.24-0.26$ & 5.39 & 48.87 \\
\hline $0.26-0.29$ & 3.36 & 52.23 \\
\hline $0.29-0.32$ & 1.82 & 54.05 \\
\hline $0.32-0.36$ & 1.07 & 55.12 \\
\hline $0.36-0.40$ & 1.05 & 56.17 \\
\hline $0.40-0.45$ & 1.5 & 57.67 \\
\hline $0.45-0.50$ & 2.01 & 59.68 \\
\hline $0.50-0.55$ & 2.29 & 61.97 \\
\hline $0.55-0.62$ & 2.3 & 64.27 \\
\hline $0.62-0.69$ & 2.32 & 66.59 \\
\hline $0.69-0.76$ & 2.59 & 69.18 \\
\hline
\end{tabular}

issues of powder agglomerates and anti-crush can be effectively resolved. Therefore the grinding efficiency has greatly improved. In the 100 hour frequency control test, transient maximums of amplitude and vibration intensity were at $16 \mathrm{~mm}$ and 18 respectively. In addition, secondary pendulum spindle strength was checked and it turned out to meet the requirements.

2) The industry wide practice shows that the new two pendulum exciter has initially solve the problem of the ultrafine powder's deaggregation and refinement. The current situation that ultrafine grinding of super hard particle is hovering over the micron level has been improved. Advancement has been achieved that the output size of diamond particles has been reduced to $\mathrm{d}(50) \leq 0.27 \mu \mathrm{m}$ with the input size being $\mathrm{d}(50) \leq 10$ $\mu \mathrm{m}$.

\section{CONFLICT OF INTEREST}

The authors confirm that this article content has no conflict of interest.

\section{ACKNOWLEDGEMENTS}

The authors acknowledge the financial support from National Natural Science Foundation of China (51375221), College students of technology innovation projects in

\begin{tabular}{c|c|c}
\hline Particle size $(\mu \mathrm{m})$ & Interval $(\%)$ & Accumulation $(\%)$ \\
\hline $0.76-0.85$ & 3.01 & 72.19 \\
\hline $0.85-0.95$ & 3.21 & 75.4 \\
\hline $0.95-1.05$ & 2.98 & 78.38 \\
\hline $1.05-1.17$ & 2.39 & 80.77 \\
\hline $1.17-1.31$ & 1.83 & 82.6 \\
\hline $1.31-1.45$ & 1.53 & 84.13 \\
\hline $1.45-1.62$ & 1.52 & 85.65 \\
\hline $1.62-1.80$ & 1.65 & 87.3 \\
\hline $1.80-2.00$ & 1.82 & 89.12 \\
\hline $2.00-2.23$ & 1.96 & 91.08 \\
\hline $2.23-2.48$ & 1.99 & 93.07 \\
\hline $2.48-2.76$ & 1.91 & 94.98 \\
\hline $2.76-3.08$ & 1.68 & 96.66 \\
\hline $3.08-3.42$ & 1.34 & 98 \\
\hline $3.42-3.81$ & 0.96 & 98.96 \\
\hline $3.81-4.24$ & 0.6 & 99.56 \\
\hline $4.24-4.72$ & 0.31 & 99.87 \\
\hline $4.72-5.25$ & 0.13 & 100 \\
\hline $5.25-5.85$ & 0 & 100 \\
\hline
\end{tabular}

Jiangsu (201511276005Z), Key Scientific and technological projects of Henan (142102210138) and Scientific and technological projects of Zhengzhou City (20130797).

\section{REFERCENCES}

[1] B.C. Wen, "Vibration Utilization Project", Machinery Industry Press, 2006.

[2] P. Huang, M.P. Jia and B.L. Zhong, "New Method to Measure the Fill Level of the Ball Mill I-Theoretical Analysis and DEM Simulation", Chinese Journal of Mechanical Engineering, vol.23, no.4, pp.460-467, 2010.

[3] Q.K. Han and Z. Luo, "Multi-body dynamics analysis, control and simulation of mechanical system", Science Press, Beijing, 2010.

[4] H.K. Jiang and X.C. Song, "Multibody dynamics simulation of balls impact-contact mechanics in ball screw mechanism", Proceedings - International Conference on Electrical and Control Engineering, pp.1320-1323, 2010.

[5] Guo Ning Tang, Meng Peng, Wei Liu, Dan Ren. Kinematic, "Characteristics Research oftheSuperfine Particles in the Superfine Vibration Mill", Applied Mechanics and Materials, 103:480-487, 2011.

[6] Xiaolan. Yang, Jifeng Liu and Luoming Zhang, "Study on chaotic vibration and high vibration intensity of a multi-level partial blocks excitation system", Journal of Vibration and Control, vol. 21, no.4, pp.627-636, 2015.

[7] Z.Y. Zhang, "MATLAB course", Beijing university of aeronautics and astronautics press, 2010 .

[8] J.X. Zhang, "UG NX/Motion basis and examples", Tsinghua University Press, Beijing, 2009.

[9] W.P. Zhang, "Mechanical Vibration Course", Metallurgical Industry Press, Beijing, 2009.

[10] Liu Jifeng. China, Patent 2,010,105040238. 2012.

(C) Xiaolan et al.; Licensee Bentham Open.

This is an open access article licensed under the terms of the (https://creativecommons.org/licenses/by/4.0/legalcode), which permits unrestricted, non-commercial use, distribution and reproduction in any medium, provided the work is properly cited. 